\title{
Produtos Alternativos na Produção de Blocos para Alvenaria Estrutural
}

\author{
Adauto José Miranda de Lima ${ }^{1}$, Setsuo Iwakiri² \\ ${ }^{1}$ Departamento Acadêmico de Construção Civil, Universidade Tecnológica Federal do Paraná - UTFPR \\ ${ }^{2}$ Departamento de Engenharia e Tecnologia Florestal, Universidade Federal do Paraná - UFPR
}

\section{RESUMO}

Este trabalho teve como objetivo avaliar o potencial de utilização do resíduo do desdobro de toras de Pinus spp., como substituição ao agregado miúdo do concreto utilizado para a produção de blocos para alvenaria estrutural. A substituição parcial do cimento Portland do concreto por sílica ativa, cinza volante e cinza de casca de arroz foi, também, analisada. A avaliação das características físicas e mecânicas do concreto foi efetuada em conformidade com as normas da ABNT. Foram testadas substituições do agregado miúdo por teores de $50 \%$ de resíduo de Pinus spp. in natura e com pré-tratamento tipo extração em água fria por 48 horas. Utilizaramse, ainda, aditivos, como superplastificante e acelerador de pega do cimento. Verificaram-se resultados satisfatórios com substituições de 50\% do agregado miúdo mineral pelo resíduo de Pinus spp. e teores de substituição do cimento Portland por 10\% de sílica ativa, 5\% de cinza volante e $30 \%$ de cinza de casca de arroz.

Palavras-chave: Resíduo de Pinus spp., alvenaria estrutural, materiais cimentícios alternativos.

\section{Pinus spp. and Cementitious Alternative Materials in the Production of Masonry Blocks}

\begin{abstract}
The purpose of this research was to evaluate the potential use of residues from sawed Pinus spp. wood, in substitution to the fine aggregate of concrete used in the production of blocks for structural masonry. The partial substitution of Portland cement in concrete by silica fume, fly ash and rice husk ash, was also analyzed. The evaluation of the physical and mechanical characteristics of the concrete was determined in agreement with the ABNT normalization. Substitutes to the fine aggregate were tested by tenors of $50 \%$ of Pinus spp. residue in natura, and then pre-treated with extraction in cold water for 48 hours. Chemical addictives (superplasticizer and accelerator) were also used. Satisfactory results were obtained with substitutions of $50 \%$ of the mineral fine aggregate by the Pinus spp. residue, $10 \%$ of silica fume, $5 \%$ of fly ash, and $30 \%$ of rice husk ash .
\end{abstract}

Keywords: Pinus spp. residue, structural masonry, cementitious alternative materials. 


\section{INTRODUÇÃO}

A viabilidade da produção de compósitos produzidos com fibras ou partículas naturais vem sendo pesquisada há décadas, possuindo uma longa história de aceitação e aplicação na construção civil, especialmente na Europa e na Ásia.

De acordo com Semple \& Evans (2004) e Moslemi $(1989,1993)$, os materiais produzidos com compósitos cimento-madeira apresentam uma série de vantagens, dentre as quais podem ser citadas: disponibilidade de matéria-prima em grandes quantidades, baixa densidade, baixa permeabilidade e possibilidade de serem serrados e de receberem parafusos e acabamentos, como pinturas e outros. Essas vantagens permitem sua utilização na produção de painéis de fechamento, forros, telhas, elementos de vedação e de alvenaria.

Além dessas vantagens, os materiais baseados em compósitos cimento-madeira não emitem resíduos tóxicos durante sua manufatura (van Elten, 2000 apud Semple \& Evans, 2004), não necessitam de tratamentos preservativos (RamirezCoretti et al.,1998) e apresentam menor dispêndio de energia no processo de produção (Moslemi, 1989), inclusive se tornando uma alternativa viável aos fibrocimentos que utilizam asbesto (amianto), principalmente na Europa, no Japão e na América do Norte (Moslemi, 1989).

A norma brasileira NBR 6136 (Associação..., 2008a) define blocos vazados para alvenaria como sendo elementos que possuem furos prismáticos perpendiculares à face que os contém e cuja seção transversal média útil seja inferior a $75 \%$ da seção transversal bruta.

Os blocos de concreto, assim como são conhecidos hoje, surgiram nos Estados Unidos da América no final do século XIX (PCA, 1988 apud Medeiros, 1993). No Brasil, existem registros da utilização de blocos de concreto por volta de 1940, sendo que atualmente inúmeros edifícios vêm sendo construídos com este material. De acordo com Sousa (2001), os blocos de concreto são materiais de grande interesse, pois, além do custo relativamente baixo, apresentam também um baixo teor energético incorporado à sua produção, na medida em que não passam pelo processo de queima, como os blocos cerâmicos para alvenaria.

Grandi (2002) e Isaia (2007) demonstram os diferenciais de um sistema construtivo tradicional, com blocos cerâmicos, em relação a outro utilizando blocos de concreto, sendo que este apresenta diversas vantagens quando comparado aos métodos convencionais, como: redução do custo da obra de até 30\%, maior velocidade na conclusão da obra e, no caso de alvenaria estrutural, dispensa o uso de vigas e pilares de concreto armado.

Normalmente, os materiais utilizados na produção de blocos de concreto se resumem em aglomerante, agregados graúdos e miúdos, água e, eventualmente, aditivos e corantes.

De acordo com Ferreira Júnior (1985), a dosagem é o estudo realizado para fixar as quantidades dos materiais constituintes, visando a obter um concreto e, consequentemente, os blocos pré-fabricados com as características desejadas, quais sejam: coesão no estado fresco - de forma que possam ser desmoldados e transportados sem que seu formato seja alterado e máxima compacidade, para que a absorção de água seja mínima e a resistência seja compatível com a aplicação a que se destinam.

O equipamento utilizado na produção de blocos de concreto é denominado vibroprensa, que recebe tal denominação em razão do mecanismo de funcionamento empregado durante o processo de moldagem dos blocos: vibração associada à prensagem. A primeira função é responsável pelo preenchimento do molde e pelo adensamento da mistura; a segunda função influencia o adensamento e o acabamento dos blocos (Sousa, 2001).

O tempo ótimo de vibração será o mínimo necessário para proporcionar aos blocos a máxima compacidade. Esse tempo irá variar conforme a máquina de moldagem e as características do concreto fresco, sendo que o importante é que seja conhecido e rigorosamente controlado, por meio de cronômetro ou de sistema automático de medição.

Depois de produzidos, os blocos devem passar por um processo de cura, que corresponde a um conjunto de operações que visa a proporcionar aos blocos, durante certo tempo, condições de umidade, temperatura e pressão necessárias a uma adequada reação de hidratação do cimento. Conforme Tango 
(1984), qualquer alteração nessas condições pode refletir diretamente nas características finais dos blocos de concreto. Mehta \& Monteiro (2008) consideram sete dias como um período mínimo de cura ao ar livre para concretos contendo cimento Portland comum.

A norma ABNT NBR 6136 (2008) estabelece os requisitos mínimos quanto às características físicas e mecânicas dos blocos de concreto simples para alvenaria estrutural e não estrutural. Conforme esta especificação técnica, para uso estrutural, o bloco deve se enquadrar no mínimo na Classe C (blocos com resistência à compressão igual ou maior do que 3,0 MPa, para uso em elementos de alvenaria acima do nível do solo).

Aïtcin (2000) e Carmo (2006) enumeram diversas vantagens da utilização de materiais cimentícios alternativos em adição ou substituição parcial ao cimento Portland, dentre as quais, a própria redução do consumo de cimento (Moslemi, 1989). O cimento é o componente mais caro de um compósito cimento-madeira e sua produção, de acordo com Oliveira (2000), é responsável por 7\% das emissões de $\mathrm{CO}_{2}$ no mundo, sendo produzida, em média, 0,8 $\mathrm{t}$ a 1,0 t de $\mathrm{CO}_{2}$ por tonelada de cimento produzido.

John \& Agopyan (1993) indicam que o emprego de materiais cimentícios alternativos pode colaborar para um aumento da durabilidade de compósitos com fibras e partículas vegetais. Tal fato se dá por meio do consumo de parte do $\mathrm{Ca}(\mathrm{OH})_{2}$ que está concentrado na zona de transição, possibilitando a melhora da aderência entre fibra e matriz, e a redução do $\mathrm{pH}$ da solução com a combinação dos íons $\mathrm{Ca}^{2+}$ presentes.

A sílica ativa é um subproduto da fabricação do silício metálico, das ligas de ferro silício e de outras ligas de silício. O silício e suas ligas são produzidos em fornos de arco elétrico imerso, em que o quartzo é reduzido na presença de carvão e ferro, durante a produção de ligas ferro-silício. Durante a redução da sílica, dentro do arco elétrico, um subóxido de silício ou monóxido de silício gasoso, $\mathrm{SiO}$, é produzido. Esse gás escapa para a parte superior da carga, se resfria, condensa e se oxida na forma de partículas finíssimas de sílica ou dióxido de silício $\left(\mathrm{SiO}_{2}\right)$, sendo estas coletadas por um sistema de eliminação de pó (Aitcin, 2000).
Conforme Carmo (2006), este rejeito industrial recebeu inicialmente denominações como fumos de sílica ou microssílica; contudo, a ABNT NBR 13956 (1997) estabeleceu a denominação sílica ativa como padrão, sendo que sua utilização já é prática consolidada na tecnologia do concreto, mas com uso ainda experimental em compósitos cimentomadeira.

Em 2001, a produção mundial de sílica ativa era de 585,7 milhões de toneladas, tendo como maiores produtores a Noruega e os Estados Unidos. No Brasil, naquele ano, a produção era de 10,5 milhões de toneladas (Instituto..., 2002 apud Carmo, 2006).

As cinzas volantes são o resíduo da queima de carvão mineral em pó, provenientes de fornos que usam carvão mineral moído como combustível. O pó, bastante nocivo ao meio ambiente, é coletado por filtros de mangas ou filtros eletrostáticos (Freitas Júnior, 2005).

De acordo com Camões (2005), a produção mundial de cinzas volantes é estimada em mais de 700 milhões de toneladas.ano ${ }^{-1}$, das quais $70 \%$ (cerca de 500 milhões de toneladas) são adequadas e podem ser utilizadas como adição em concretos e outros produtos de cimento.

A utilização de cinzas volantes pode resultar em um retardamento da velocidade do ganho de resistência mecânica nas primeiras idades dos compósitos cimentícios (até os 28 dias); porém, posteriormente, a resistência alcançada se iguala e até ultrapassa a do clínquer hidratado, segundo Mehta \& Monteiro (2008).

No Brasil, a maior fração do uso dessas adições é na composição dos cimentos Portland tipos CP II Z - cimento Portland composto com adição de pozolana, conforme norma ABNT NBR 11578 (Associação..., 1991c), e CP IV - cimento Portland pozolânico, conforme a norma ABNT NBR 5736 (Associação..., 1991b)

A casca de arroz é uma capa lenhosa do grão, dura, com alto teor de sílica, composta de aproximadamente $50 \%$ de celulose, $30 \%$ de lignina e 20\% de sílica de base anidra (Mehta, 1992 apud Santos, 1997). Quando queimada, como fonte de calor para o processo de secagem e parboilização dos grãos, a lignina e a celulose são removidas, resultando numa estrutura celular porosa. 
Conforme Pouey (2006), após a queima completa, cerca de $20 \%$ da casca de arroz é convertida em cinzas, as quais ainda são consideradas um resíduo agroindustrial sem destinação certa, sendo simplesmente descartadas ou lançadas em aterros, rios, etc., gerando assim muitos problemas ambientais.

A vantagem do uso da Cinza de Casca de Arroz (CCA) é que a fonte agrícola é farta. Dos 12,1 milhões de toneladas de arroz que o País produz por ano (Instituto..., 2009), a casca corresponde a $20 \%$ dessa massa (2,4 milhões de toneladas). Esse resíduo agrícola poderia se converter em 480 mil toneladas de sílica, o suficiente para suprir o mercado de concretos estruturais e outros. Dessa forma, se essa tecnologia fosse aplicada integralmente, poder-se-ia resolver um grave problema ambiental e gerar uma fonte de renda adicional para os produtores de arroz.

O efetivo emprego da CCA na construção civil encontra restrições por alguns motivos, como sua coloração escura e a falta de uniformidade apresentada em termos de características mineralógicas. A cor escura não é um problema de ordem técnica, mas sim estética, que pode ser contornado mediante tratamentos químicos, tal como banho com ácido clorídrico $(\mathrm{HCl})$, que permite o clareamento das cinzas até a cor branca (Dafico \& Prudêncio Júnior, 2002).

Em função da grande finura, a utilização tanto da sílica ativa quanto da cinza de casca de arroz, em substituição de parte do cimento, resulta em um aumento na demanda de água para manter a mesma trabalhabilidade de uma pasta produzida com cimento puro. Produz-se, dessa forma, um compósito com relação água/cimento mais elevada. Assim, todo o benefício que se consegue na resistência e na durabilidade do compósito pela ação físico-química da sílica ativa e da CCA pode ser contraposto pela maior quantidade de água necessária para tornar o material trabalhável (Almeida, 1996 apud Mendes, 2002).

Para evitar-se a adição de mais água na mistura, é imprescindível o uso de aditivos superplastificantes, aditivos redutores de água de alta eficiência, que dispersam as partículas de cimento e sílica ativa, diminuindo o atrito interno existente entre elas
(Khayat \& Aïtcin, 1993 apud Mendes, 2002; Mehta \& Monteiro, 2008; Neville, 1997).

Os aceleradores de pega, por sua vez, são compostos orgânicos ou inorgânicos solúveis em água e são utilizados para propiciar uma redução do tempo de cura, um aumento da velocidade de desenvolvimento da resistência inicial do material (Mehta \& Monteiro, 2008).

Conforme Isaia (2005), dentre os compostos inorgânicos, encontram-se os sais de elementos alcalinos ou alcalinoterrosos à base de cloretos, fluoretos, carbonatos, silicatos, fluorsilicatos, aluminatos, boratos, nitratos, nitritos, tiossulfatos, etc. Para Mehta \& Monteiro (2008), o aditivo acelerador de pega mais conhecido e amplamente utilizado é o cloreto de cálcio $\left(\mathrm{CaCl}_{2}\right)$.

\section{MATERIAL E MÉTODOS}

\subsection{Materiais utilizados}

O resíduo de madeira de Pinus spp. utilizado neste estudo foi coletado na empresa Madeireira Juruqui, localizada no Município de Almirante Tamandaré-PR. Após a coleta, o resíduo foi seco ao ar e peneirado em uma peneira $A B N T n^{\circ} 4$, de forma a garantir uma granulometria contínua com dimensão máxima característica (Dmáx) de 4,8 mm.

$\mathrm{O}$ aglomerante utilizado na pesquisa foi $\mathrm{o}$ Cimento Portland, tipo CPV ARI RS - cimento Portland de Alta Resistência Inicial - Resistente a Sulfatos, especificado pela norma ABNT NBR 5733 (Associação..., 1991a).

Os materiais cimentícios alternativos utilizados foram a sílica ativa produzida pela Camargo Corrêa S.A., com nome comercial de Silmix, a cinza volante oriunda da queima do carvão mineral da Usina Termelétrica de Charqueadas-RS e a cinza de casca de arroz fornecida por uma empresa de beneficiamento de arroz do município de Jaraguá do Sul-SC. Os agregados minerais convencionais foram obtidos na região de Curitiba-PR.

Os aditivos químicos utilizados foram o acelerador de pega cloreto de cálcio bi-hidratado $\left(\mathrm{CaCl}_{2} \cdot 2 \mathrm{H}_{2} \mathrm{O}\right)$ e o superplastificante de Pega Normal 
Muraplast FK 49B, produzido pela empresa MC Bauchemie Brasil Indústria e Comércio Ltda.

Os agregados minerais convencionais e o resíduo de Pinus spp. utilizados, oriundos da região de Curitiba-PR, apresentam as características constantes da Tabela 1 .

\subsection{Metodologia}

Em um estudo preliminar, foram produzidas três séries de blocos de concreto, com traços 1:6, 1:8 e 1:10, ou seja, uma parte de cimento para seis, oito e dez partes de agregados secos. Na medida em que todos apresentaram desempenho satisfatório para utilização em alvenaria estrutural, ficou definido, principalmente em função das características econômicas, o traço 1:10 como Referência para este estudo.

Os blocos de concreto foram produzidos em uma máquina vibroprensa marca Trillor Montana, modelo $M B X$ 200. Esse equipamento (Figura 1) infere uma carga unidirecional vertical de $1.010 \mathrm{kgf}$, distribuída durante a compressão do concreto fresco para produção de três blocos de cada vez, tendo capacidade de produzir até 4 mil blocos por dia.

A proporção do agregado total (agregado miúdo + agregado graúdo) foi feita mediante a determinação da mistura de maior massa unitária compactada, o que traduz o menor índice de vazios possível para a mistura, conforme método da Associação Brasileira

Tabela 1. Características do resíduo de Pinus spp. e dos agregados minerais convencionais.

Table 1. Characteristics of the residues of Pinus spp. and the conventional mineral aggregates.

\begin{tabular}{|c|c|c|c|c|}
\hline Características & Norma & $\begin{array}{l}\text { Ag. } \\
\text { miúdo }\end{array}$ & $\begin{array}{c}\text { Ag. } \\
\text { graúdo }\end{array}$ & $\begin{array}{l}\text { Resíduo de } \\
\text { Pinus spp. }\end{array}$ \\
\hline Massa específica $\left(\mathrm{g} \cdot \mathrm{cm}^{-3}\right)$ & NBR NM 52 e 53 (2003) & 2,64 & 2,70 & 1,28 \\
\hline Massa unitária $\left(\mathrm{g} \cdot \mathrm{cm}^{-3}\right)$ & NBR NM 52 e 53 (2003) & 1,56 & 1,52 & 0,17 \\
\hline Densidade madeira sólida $\left(\mathrm{g} \cdot \mathrm{cm}^{-3}\right)$ & NBR 7190 (1997) & & & 0,41 \\
\hline Dimensão máxima (mm) & NBR NM 248 (2008) & 4,80 & 9,50 & 4,80 \\
\hline Módulo de finura & NBR NM 248 (2008) & 2,92 & 5,67 & 3,25 \\
\hline Teor mat. pulverulento (\%) & NBR NM 46 (2003) & 1,08 & 0,25 & 0,27 \\
\hline Agregado miúdo (\%) & NBR NM 248 (2008) & 96,82 & 7,05 & 99,73 \\
\hline Agregado graúdo (\%) & NBR NM 248 (2008) & 2,10 & 92,70 & 0,00 \\
\hline Teor argila em torrões (\%) & NBR NM 7218 (1987) & 0,00 & 0,00 & 0,00 \\
\hline Teor mat. orgânica (ppm) & NBR NM NM 49 (2001) & $<100,00$ & $<100,00$ & - \\
\hline Absorção de água (\%) & NBR NM NM 30 (2001) & 1,47 & 0,80 & 129,62 \\
\hline Coef. inchamento médio & NBR NM 6467 (2006) & 1,44 & - & 7,79 \\
\hline Teor umidade crítica (\%) & NBR NM 6467 (2006) & 5,00 & - & - \\
\hline Extrativos totais (\%) & TAPPI T204cm (1997) & - & - & 2,42 \\
\hline $\mathrm{pH}$ & TAPPI T204cm (1997) & - & - & 7,79 \\
\hline Classificação & NBR 7211 (2008) & $\begin{array}{c}\text { Areia } \\
\text { Zona }-3 \mathrm{ABNT}\end{array}$ & Brita 0 & $\begin{array}{c}\text { Equivalente } \\
\text { Zona-3 ABNT }\end{array}$ \\
\hline
\end{tabular}
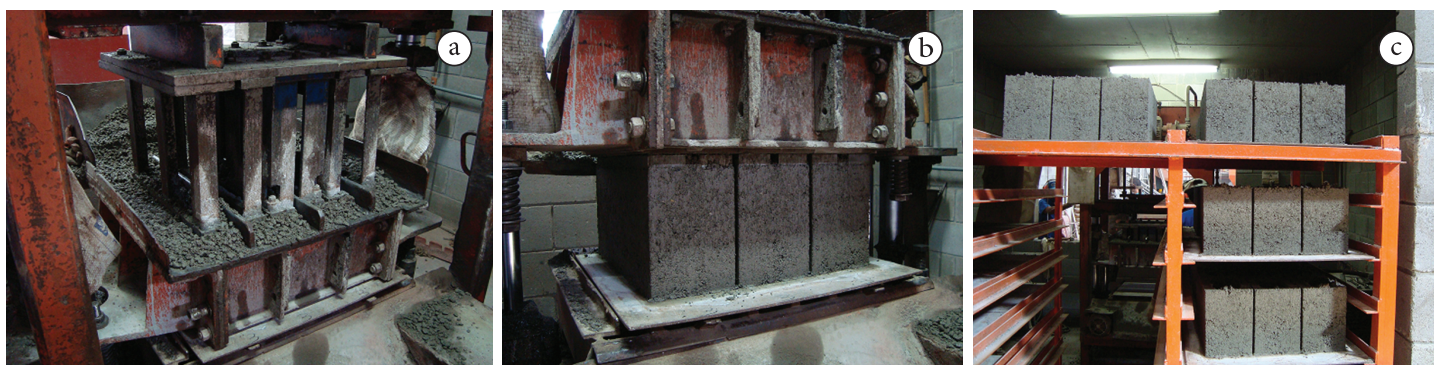

Figura 1. Produção de blocos protótipos: a) vibrocompactação do concreto; b) abertura dos extratores; c) cura dos blocos.

Figure 1. Production of prototypes blocks: a) vibrate-compression of concrete; b) opening of the extractors; c) curing of the blocks 
de Cimento Portland (ABCP, 1985). A umidade de moldagem, após testes de moldagem com a máquina produtora de blocos, foi fixada em $6 \%$ (em relação à soma das massas dos materiais secos, inclusive do cimento Portland). A composição do concreto Traço 1:10 ficou estabelecida conforme Tabela 2.

$\mathrm{Na}$ produção dos concretos com substituição parcial do agregado miúdo convencional, a areia foi substituída, conforme estudos anteriores de Lima (2009), por 50\% de resíduo de Pinus spp. nas seguintes formas: in natura, ou seja, sem qualquer pré-tratamento ou uso de aditivos químicos; in natura e com $4,5 \% \mathrm{de} \mathrm{CaCl}_{2} \cdot 2 \mathrm{H}_{2} \mathrm{O}$; com pré-tratamento tipo extração em água fria por 48 horas $(\mathrm{AF}-48 \mathrm{H})$ e com $4,5 \%$ de $\mathrm{CaCl}_{2} \cdot 2 \mathrm{H}_{2} \mathrm{O}$.

Os teores de substituição do cimento Portland do concreto por $10 \%$ de sílica ativa, $5 \%$ de cinza volante e $30 \%$ de cinza de casca de arroz tiveram por base estudos com compósitos, efetuados anteriormente pelos autores e apresentados em Lima (2009).

Tabela 2. Composição do concreto traço 1:10 em massa $\left(1 \mathrm{~m}^{3}\right)$

Table 2. Composition of concrete mix 1:10 in mass $\left(1 \mathrm{~m}^{3}\right)$.

\begin{tabular}{|lc|}
\hline \multicolumn{1}{|c}{ Traço em volume } & $\mathbf{1}: \mathbf{1 0}$ \\
\hline \multicolumn{1}{|c}{ Materiais } & Massa $(\mathbf{k g})$ \\
\hline Cimento & 220,00 \\
\hline Agregado total & $2.119,10$ \\
\hline Areia $-50 \%$ & $1.073,31$ \\
Brita $0-50 \%$ & $1.045,79$ \\
\hline Água - umidade de Moldagem & - \\
\hline U $=6,0 \%$ & 140,35 \\
\hline
\end{tabular}

Os teores de substituição do agregado miúdo convencional, bem como os pré-tratamentos ao resíduo de Pinus spp. e os teores de aditivos químicos utilizados nos concretos para blocos são apresentados na Tabela 3.

A mistura dos materiais foi efetuada em uma betoneira de eixo horizontal com capacidade de $160 \mathrm{~L}$ e o teor de água da mistura foi controlado e corrigido em função do teor de umidade do resíduo de Pinus spp., de forma a manter sempre a madeira com o teor de umidade em $30 \%$.

O número de repetições mínimo usado, a fim de garantir estatisticamente a distribuição Normal e a confiabilidade dos resultados, foi determinado pela Equação 1:

$n=z_{\alpha / 2}^{2} \cdot \frac{C V^{2}}{E r^{2}}$

em que: $\mathrm{n}=$ número de repetições; $\mathrm{Er}=$ erro relativo admitido da estimativa, fixado em $10 \%$; $\mathrm{CV}$ = coeficiente de variação da amostra; $\mathrm{z}_{\alpha / 2}=$ valor tabelado que, para $\alpha=5 \%$ de significância, possui o valor de 1,96 .

Após a produção dos blocos protótipos, estes permaneceram em cura no mesmo local onde foram moldados, por um período de três dias.

As características físicas (densidade, índice de vazios, absorção de água e retração por secagem) foram determinadas conforme norma ABNT NBR 9778 (Associação..., 2006b).

Tabela 3. Teores de substituição do agregado convencional, pré-tratamentos ao resíduo de Pinus spp. e teores de aditivos químicos e de materiais cimentícios alternativos.

Table 3. Tenors of substitution of the conventional fine aggregate, pré-treatments to the residue of Pinus spp., tenors of chemical addictives and alternative cimentitious materials.

\begin{tabular}{lccccc} 
Compósito & $\begin{array}{c}\text { Pré- } \\
\text { tratamento }\end{array}$ & $\begin{array}{c}\text { Substituição do agregado } \\
\text { miúdo convencional } \\
\text { (em volume) }\end{array}$ & $\begin{array}{c}\text { Aditivo } \\
\text { acelerador } \\
\mathbf{C a C l}_{2} \cdot \mathbf{2} \mathbf{H}_{2} \mathbf{O}\end{array}$ & $\begin{array}{c}\text { Substituição ao } \\
\text { cimento Portland } \\
\text { (tipo e teor) }\end{array}$ & $\begin{array}{c}\text { Aditivo } \\
\text { superplastificante }\end{array}$ \\
\hline $\mathrm{PN}^{2}$ & in natura & $50 \%$ & - & - & - \\
$\mathrm{PNCC}^{3}$ & in natura & $50 \%$ & $4,5 \%$ & - & - \\
PNAFCC $^{4}$ & $\mathrm{AF}-48 \mathrm{H}$ & $50 \%$ & $4,5 \%$ & - & - \\
PNAF SA $^{5}$ & $\mathrm{AF}-48 \mathrm{H}$ & $50 \%$ & - & Sílica ativa $10 \%$ & $0,6 \%$ \\
PNAFCV $^{6}$ & $\mathrm{AF}-48 \mathrm{H}$ & $50 \%$ & - & $\begin{array}{c}\text { Cinza volante } \\
5 \%\end{array}$ & - \\
PNAFCCA $^{7}$ & $\mathrm{AF}-48 \mathrm{H}$ & $50 \%$ & - & $\begin{array}{c}\text { Cinza de sasca de } \\
\text { arroz } 30 \%\end{array}$ & $2,0 \%$ \\
\hline
\end{tabular}


A determinação da resistência e da condutividade térmica foi feita por meio de técnicas fluxométricas, utilizando um Condutivímetro Fluxométrico, conforme normas ABNT NBR 15.220-4 (2005), em consonância com as normas internacionais ISO/ DP 8302 (International..., 1991) e ASTM C177 (American..., 2008a).

As características mecânicas dos blocos protótipos, compreendendo os ensaios para determinação da resistência à compressão aos três dias (RC 3d), sete dias (RC 7d) e 28 dias (RC 28d), foram determinadas conforme ABNT NBR 12118 (Associação..., 2008c). O equipamento utilizado foi uma máquina universal de ensaio marca Emic, modelo PC 200.

A determinação da resistência à tração do concreto para blocos foi determinada por meio de compressão diametral de corpos de prova cilíndricos, conforme a norma ABNT NBR 7222 (Associação..., 1994). Para tanto, os corpos de prova, com diâmetro nominal de $50 \mathrm{~mm}$, foram moldados em uma única camada e adensados pelo efeito concomitante de vibração e compressão, de forma a reproduzir a moldagem efetuada em uma máquina produtora de blocos (vibroprensa). O aparato para moldagem, baseado na norma ASTM C 1170 (American..., 2008b) - Standard Test Methods for Determining Consistency and Density of Roller-Compacted Concrete Using a Vibrating Table, foi desenvolvido pelo autor desta pesquisa.

O equipamento desenvolvido (Figura 2a) é composto de cinco pistões de aço dispostos sobre uma mesa vibratória. Cada pistão, com massa de $10,5 \mathrm{~kg}$, desliza por um tubo guia e compacta o concreto no interior de um molde cilíndrico de $5 \mathrm{~cm}$ de diâmetro e $10 \mathrm{~cm}$ de altura. Para garantir que o molde seria inteiramente preenchido, produziu-se um prolongamento para os moldes dos corpos de prova, confeccionados em PVC (Figuras 2b e 2c).

O tempo de vibração e compactação do concreto foi determinado em função do tempo necessário para produção de um concreto fresco com a mesma massa carcaterística do concreto produzido pela vibroprensa comercial. Esse tempo, denominado de tempo equivalente de moldagem, ou tempo Vebe, foi determinado, para este experimento, em 27 segundos.

Para a desmoldagem e a cura do concreto produzido com os CP cilíndricos, seguiram-se os procedimentos indicados na ABNT NBR 5738 (Associação..., 2003e). A cura inicial dos corpos de prova, com duração de 24 horas, deu-se em câmara úmida, conforme ABNT NBR 9479 (Associação..., 2006a), com temperatura de $23 \pm 2{ }^{\circ} \mathrm{C}$ e umidade relativa mínima de $95 \%$, quando permaneceram com os moldes e cobertos com uma placa de acrílico para proteção, conforme ABNT NBR 7215 (Associação..., 1997b). Após o período de cura inicial, os corpos de prova foram desmoldados, identificados e mantidos em câmara úmida, imersos em água, até a data de ensaio.

A análise estatística dos resultados obtidos nos ensaios foi feita por meio da análise variância (ANOVA) e, todas as vezes em que a hipótese da nulidade foi rejeitada, efetuou-se a comparação das médias por meio do teste de Tukey-Kramer a 95\% de probabilidade.


Figura 2. Sistema alternativo de moldagem por vibrocompressão: a) composição do sistema; b) preenchimento dos corpos de prova cilindricos; c) acabamento da superfície dos corpos de prova.

Figure 2. Alternative system of molding for vibrate-compression: a) composition of the system; b) completion of the cylindrical specimen; c) finish of the cylindrical specimens surface. 


\section{RESULTADOS E DISCUSSÃO}

\subsection{Características físicas}

As características físicas determinadas para os blocos protótipos e de referência são apresentadas na Tabela 4.

Analisando-se os valores da Tabela 4, constatase redução significativa da densidade dos blocos produzidos com a substituição parcial de 50\% em volume do agregado miúdo mineral pelo resíduo de Pinus spp., independentemente das substituições parciais do cimento Portland. Tais reduções variaram entre 9,23 e $12,77 \%$, em comparação com os blocos produzidos com concreto convencional.

Com relação à porosidade do concreto, verificase que o valor médio do índice de vazios do concreto com resíduo de Pinus spp. apresentou aumentos significativos quando comparado com o concreto convencional, da ordem de 15,10\%. A exceção se verificou para os blocos produzidos com $50 \%$ de Pinus spp. AF48H com substituição de $10 \%$ do cimento Portland por Sílica Ativa, que apresentou redução do índice de vazios de 6,27\%. Tal redução se deve à extrema finura da Sílica Ativa, cujas partículas esféricas, conforme dados do fabricante, apresentam diâmetros entre 0,1 e $2 \mu \mathrm{m}$, e superfície específica da ordem de $220.000,00 \mathrm{~cm}^{2} \cdot \mathrm{g}^{-1}$.
A absorção de água determinada para os blocos protótipos apresentou aumentos entre 3,89 e $45,19 \%$ em relação à absorção verificada para os blocos produzidos com o concreto convencional. O menor valor, como esperado, foi verificado para o bloco protótipo produzido com $50 \%$ de Pinus spp. AF48H com substituição parcial de $10 \%$ do cimento Portland por Sílica Ativa, visto apresentar a menor porosidade. É importante salientar que, apesar dos valores mais altos determinados para a absorção de água, todos os blocos protótipos apresentaram valores abaixo dos limites estabelecidos pela norma ABNT NBR 6136 (Associação..., 2008a).

Quanto à retração por secagem, os valores médios determinados ficaram $8,74 \%$ acima dos determinados para os blocos produzidos com concreto convencional (Figura 3). Apesar dessa variação, os valores determinados se apresentaram menores que o limite de $0,065 \%$, estabelecido pela norma ABNT NBR 6136 (Associação..., 2008a). Essa característica é de suma importância, pois a variação dimensional dos blocos acima dos limites referidos poderia ser a fonte causadora de fissuras de origem higrotérmica nas paredes das edificações (Tango, 1984).

Os valores de condutividade térmica dos materiais com os quais os blocos protótipos foram produzidos estão apresentados na Tabela 5 .

Como pode ser verificado, a substituição de 50\% em volume do agregado miúdo convencional pelo resíduo de Pinus spp. fez com que a condutividade

Tabela 4. Características físicas dos blocos protótipos.

Table 4. Physical characteristics of prototypes blocks.

\begin{tabular}{|c|c|c|c|c|c|c|c|c|}
\hline \multirow{2}{*}{$\begin{array}{c}\text { Blocos } \\
\text { composição }\end{array}$} & \multicolumn{2}{|c|}{ DE } & \multicolumn{2}{|c|}{ IV } & \multicolumn{2}{|c|}{ ABS } & \multicolumn{2}{|c|}{ RS } \\
\hline & $\begin{array}{c}\text { Média } \\
\left(\mathrm{kg} / \mathrm{m}^{3}\right)\end{array}$ & $\begin{array}{l}\text { CV } \\
(\%)\end{array}$ & $\begin{array}{c}\text { Média } \\
(\%)\end{array}$ & $\begin{array}{l}\text { CV } \\
\text { (\%) }\end{array}$ & $\begin{array}{c}\text { Média } \\
(\%)\end{array}$ & $\begin{array}{l}\text { CV } \\
(\%)\end{array}$ & $\begin{array}{c}\text { Média } \\
(\%)\end{array}$ & $\begin{array}{l}\text { CV } \\
\text { (\%) }\end{array}$ \\
\hline Concreto T $1: 10^{1}$ & $2.242,36^{\mathrm{A}}$ & 0,85 & $12,12^{\mathrm{D}}$ & 1,88 & $5,40^{\mathrm{D}}$ & 2,71 & $0,0446^{\mathrm{BC}}$ & 13,99 \\
\hline $\mathrm{PN}^{2}$ & $2.019,38^{\mathrm{BC}}$ & 1,95 & $14,54^{\mathrm{B}}$ & 5,49 & $7,21^{\mathrm{B}}$ & 7,36 & $0,0422^{\mathrm{C}}$ & 4,06 \\
\hline $\mathrm{PNCC}^{3}$ & $1.977,97^{\mathrm{BC}}$ & 1,42 & $15,51^{\mathrm{A}}$ & 3,19 & $7,84^{\mathrm{A}}$ & 2,21 & $0,0446^{\mathrm{BC}}$ & 9,14 \\
\hline PNAFCC $^{4}$ & $2.035,34^{\mathrm{B}}$ & 0,72 & $13,47^{\mathrm{C}}$ & 1,36 & $6,62^{\mathrm{C}}$ & 1,76 & $0,0443^{\mathrm{BC}}$ & 5,43 \\
\hline PNAF SA $^{5}$ & $2.028,61^{\mathrm{BC}}$ & 6,49 & $11,36^{\mathrm{D}}$ & 7,80 & $5,61^{\mathrm{D}}$ & 6,03 & $0,0528^{\mathrm{AB}}$ & 10,08 \\
\hline PNAFCV $^{6}$ & $1.956,08^{\mathrm{C}}$ & 1,36 & $14,53^{\text {B }}$ & 0,98 & $7,43^{\mathrm{B}}$ & 1,07 & $0,0526^{\mathrm{AB}}$ & 5,61 \\
\hline PNAFCCA $^{7}$ & $1.964,29^{\mathrm{BC}}$ & 1,66 & $14,62^{\mathrm{B}}$ & 3,71 & $7,45^{\mathrm{B}}$ & 3,58 & $0,0545^{\mathrm{A}}$ & 15,66 \\
\hline
\end{tabular}

Letras diferentes denotam diferenças estatísticas entre as médias nas colunas no nível de $95 \%$ de confiança; ME: Massa Específica; IV: Índice de Vazios; ABS: Absorção de Água: RS: Retração por Secagem; CV: Coeficiente de Variação; Concreto T 1:101: Concreto convencional para blocos traço 1:10; $\mathrm{PN}^{2}: 50 \%$ de Pinus spp. in natura; $\mathrm{PNCC}^{3}: 50 \%$ de Pinus spp. in natura $+4,5 \% \mathrm{CaCl}_{2} ; \mathrm{PNAFCC}^{4}$ $50 \%$ de Pinus spp. in natura com 48 horas de tratamento em água fria $+4,5 \% \mathrm{CaCl}_{2}$; PNAFSA 5 : $50 \%$ de Pinus spp. in natura com 48 horas de tratamento em água fria $+10 \%$ de Sílica Ativa e $0,60 \%$ de Superplastificante; PNAFCV 6 : 50\% de Pinus spp. in natura com 48 horas de tratamento em água fria $+5 \%$ de Cinza Volante; PNAFCCA 7 : $50 \%$ de Pinus spp. in natura com 48 horas de tratamento em água fria $+30 \%$ de Cinza de Casca de Arroz $+2,2 \%$ de Superplastificante. 


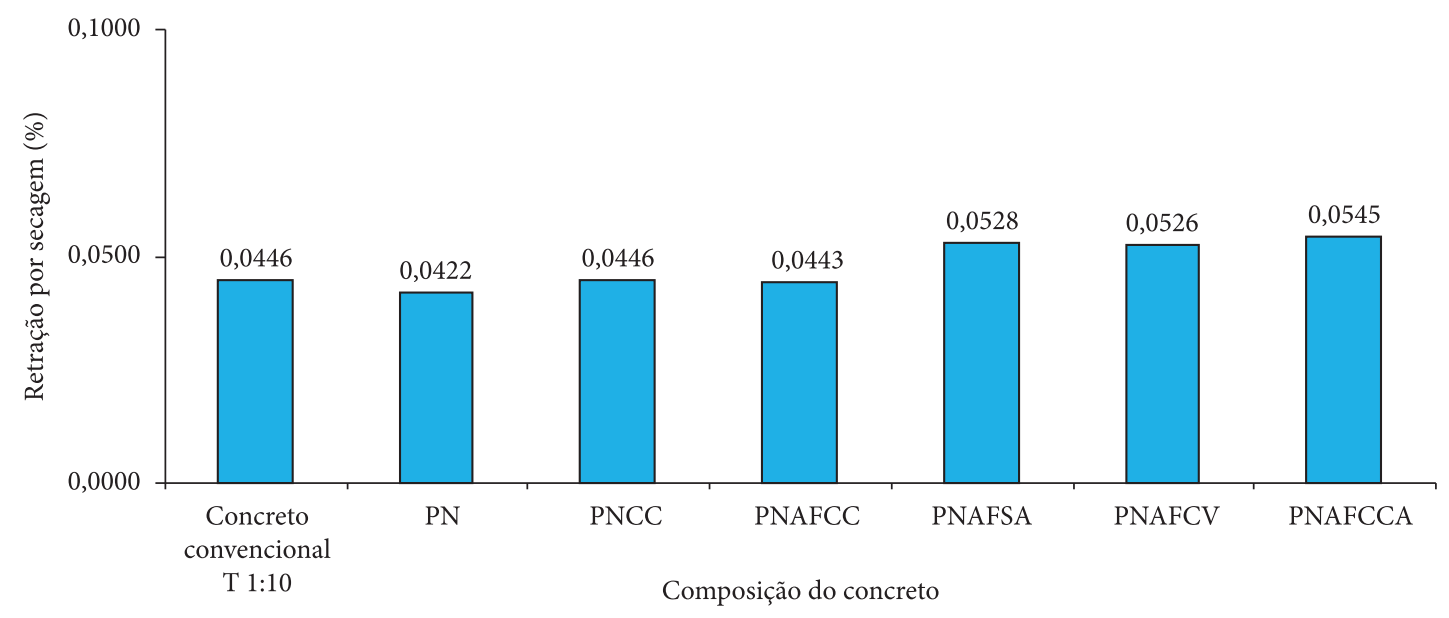

Figura 3. Retração por secagem.

Figure 3. Drying shrinkage.

Tabela 5. Condutividade térmica dos blocos protótipos.

Table 5. Thermal conductivity of prototypes blocks.

\begin{tabular}{lccc} 
Blocos composição & Condutividade térmica $\left(\mathbf{W} \cdot \mathbf{m K}^{-1}\right)$ & CV (\%) & Variação (\%) \\
\hline Concreto T $1: 10^{1}$ & $0,90^{\mathrm{A}}$ & 0,64 & - \\
PN $^{2}$ & $0,61^{\mathrm{CD}}$ & 9,13 & $-32,22$ \\
PNCC $^{3}$ & $0,64^{\mathrm{BC}}$ & 2,40 & $-28,89$ \\
PNAFCC $^{4}$ & $0,60^{\mathrm{CD}}$ & 3,38 & $-33,33$ \\
PNAF SA $^{5}$ & $0,68^{\mathrm{B}}$ & 7,37 & $-24,44$ \\
PNAFCV $^{6}$ & $0,57^{\mathrm{DE}}$ & 6,08 & $-36,67$ \\
PNAFCCA $^{7}$ & $0,52^{\mathrm{E}}$ & 5,09 & $-42,22$ \\
\hline
\end{tabular}

Letras diferentes denotam diferenças estatísticas entre as médias nas colunas no nível de $95 \%$ de confiança; CV: Coeficiente de Variação; Concreto T 1:101: Concreto convencional para blocos traço 1:10; $\mathrm{PN}^{2}: 50 \%$ de Pinus spp. in natura; PNCC $: 50 \%$ de Pinus spp. in natura $+4,5 \% \mathrm{CaCl}_{2} ; \mathrm{PNAFCC}^{4}: 50 \%$ de Pinus spp. in natura com 48 horas de tratamento em água fria $+4,5 \% \mathrm{CaCl}_{2} ; \mathrm{PNAFSA}^{5}$ : $50 \%$ de Pinus spp. in natura com 48 horas de tratamento em água fria $+10 \%$ de Sílica Ativa e 0,60\% de Superplastificante; PNAFCV ${ }^{6}$ $50 \%$ de Pinus spp. in natura com 48 horas de tratamento em água fria $+5 \%$ de Cinza Volante; PNAFCCA ${ }^{7}: 50 \%$ de Pinus spp. in natura com 48 horas de tratamento em água fria $+30 \%$ de Cinza de Casca de Arroz + 2,2\% de Superplastificante.

térmica média do concreto para blocos traço 1:10 fosse reduzida de $0,90 \mathrm{~W} \cdot \mathrm{mK}^{-1}$ para valores em torno de 0,52 a 0,68 W.mK $\mathrm{mK}^{-1}$, portanto com reduções significativas da ordem de 24,44 a 42,22\% em comparação à condutividade térmica apresentada pelo concreto convencional para blocos, traço $1: 10$; tal fato corrobora a tese de que os materiais produzidos com base em compósitos cimentomadeira apresentam características de isolamento térmico superiores aos materiais cimentícios convencionais. Os valores de condutividade térmica dos blocos protótipos e de referência são apresentados na Figura 4.

\subsection{Características mecânicas}

As médias de resistências à compressão aos 3, 7 e 28 dias de cura, determinadas para os blocos protótipos, e de referência são apresentadas na Tabela 6 e na Figura 5.

Como pode ser verificado, aos três dias de cura, os blocos protótipos apresentaram reduções da resistência à compressão, em comparação com os blocos produzidos com concreto convencional, variando entre 36,23 e 55,93\%. Aos sete dias de cura, a redução das resistências variou entre 56,42 e $64,91 \%$, e aos 28 dias de cura, reduções entre 56,74 e $66,39 \%$. Apesar de tais reduções serem significativas, todos os blocos atingiram a resistência mínima para utilização com finalidade estrutural, cuja resistência mínima, estabelecida pela norma ABNT NBR 6136 (Associação..., 2008a), é de 3,0 MPa. De acordo com os resultados verificados aos 28 dias de cura, os blocos protótipos podem ser utilizados como 




Figura 4. Condutividade térmica.

Figure 4. Thermal conductivity.

Tabela 6. Resistência à compressão dos blocos protótipos.

Table 6. Compression strength of prototypes blocks.

\begin{tabular}{lcccccc}
\multicolumn{1}{c}{$\begin{array}{c}\text { Blocos } \\
\text { composição }\end{array}$} & \multicolumn{7}{c}{ Resistência à compressão } \\
\cline { 2 - 7 } Concreto T $1: 10^{1}$ & M3d (MPa) & $\mathbf{C V}(\%)$ & M7d (MPa) & $\mathbf{C V}(\%)$ & $\mathbf{M} 28 d(\mathbf{M P a})$ & $\mathbf{C V}(\%)$ \\
PN $^{2}$ & $5,99^{\mathrm{A}}$ & 6,98 & $10,83^{\mathrm{A}}$ & 2,60 & $13,06^{\mathrm{A}}$ & 3,86 \\
PNCC $^{3}$ & $2,64^{\mathrm{E}}$ & 6,48 & $3,80^{\mathrm{E}}$ & 7,20 & $4,39^{\mathrm{E}}$ & 8,68 \\
PNAFCC $^{4}$ & $3,35^{\mathrm{D}}$ & 7,08 & $4,21^{\mathrm{CD}}$ & 6,07 & $4,61^{\mathrm{DE}}$ & 3,58 \\
PNAF SA $^{5}$ & $3,48^{\mathrm{CD}}$ & 4,27 & $4,57^{\mathrm{BC}}$ & 4,53 & $5,16^{\mathrm{C}}$ & 7,19 \\
PNAFCV $^{6}$ & $3,82^{\mathrm{B}}$ & 3,08 & $4,48^{\mathrm{BCD}}$ & 5,43 & $5,03^{\mathrm{CD}}$ & 4,22 \\
PNAFCCA $^{7}$ & $3,77^{\mathrm{BC}}$ & 6,16 & $4,72^{\mathrm{B}}$ & 6,79 & $5,65^{\mathrm{B}}$ & 4,58 \\
\hline
\end{tabular}

Letras diferentes denotam diferenças estatísticas entre as médias nas colunas no nível de 95\% de confiança; CV: Coeficiente de Variação; Concreto T 1:101: Concreto convencional para blocos traço 1:10; PN² $^{2}$ 50\% de Pinus spp. in natura; PNCC $30 \%$ de Pinus spp. in natura $+4,5 \% \mathrm{CaCl}_{2}$; PNAFCC ${ }^{4}: 50 \%$ de Pinus spp. in natura com 48 horas de tratamento em água fria $+4,5 \% \mathrm{CaCl}_{2}$; PNAFSA $^{5}$ : 50\% de Pinus spp. in natura com 48 horas de tratamento em água fria $+10 \%$ de Sílica Ativa e $0,60 \%$ de Superplastificante; PNAFCV 6 : 50\% de Pinus spp. in natura com 48 horas de tratamento em água fria + 5\% de Cinza Volante; PNAFCCA $: 50 \%$ de Pinus spp. in natura com 48 horas de tratamento em água fria $+30 \%$ de Cinza de Casca de Arroz $+2,2 \%$ de Superplastificante. $M=$ Valor médio.

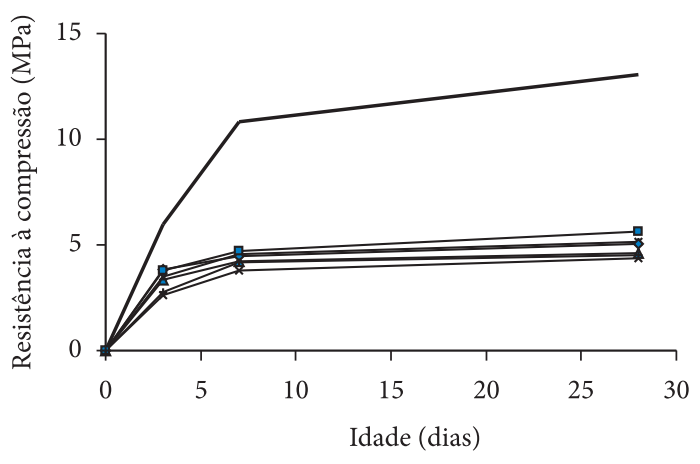

$\begin{array}{ll}\text { - Concreto convencional T } 1: 10 & \text { * PN } \\ \rightarrow-\text { PNCC } & \text { - PNAFCC } \\ \rightarrow \text { PNAF SA } & - \text { PNAF CV } \\ \rightarrow \text { PNAF CCA } & \end{array}$

Figura 5. Evolução da resistência à compressão com a idade.

Figure 5. Evolution of compression strength with age. elementos estruturais e de vedação na construção de edificações de 4 a 5 pavimentos, tais como as edificações de interesse social, alvo principal da aplicação do material desenvolvido.

O gráfico apresentado na Figura 3 indica que apesar das reduções significativas das resistências apresentadas pelos blocos protótipos em comparação aos blocos de referência, em todas as idades, houve ganhos importantes de resistência dos blocos protótipos ao longo do tempo, sendo que essas médias aumentaram, em média, 49,44\% entre o terceiro e o $28^{\circ}$ dia de cura. A inclinação ascendente dos gráficos apresentados na Figura 3 indica, ainda, a possibilidade de continuidade de ganhos de resistência dos blocos protótipos ao longo do tempo. 
As resistências médias à tração do concreto utilizado para a produção dos blocos protótipos e de referência, determinadas por compressão diametral em corpos de prova cilíndricos, aos 28 dias de cura, conforme a norma ABNT NBR 7222 (Associação..., 1994), são apresentadas na Tabela 7.

Os resultados indicam uma variação da resistência à tração do concreto para produção de blocos, traço 1:10, que variam desde uma redução de $28,90 \%$ - no caso do concreto produzido com substituição do agregado miúdo mineral do concreto por 50\%, em volume, de resíduo de Pinus spp. in natura - até incremento de 5,78\%, como o verificado para o concreto com $50 \%$ de resíduo de Pinus spp. AF48H com substituição de $30 \%$ do cimento Portland por cinza de casca de arroz (CCA). Os valores determinados para os protótipos foram muito maiores que o esperado, visto que a relação entre a resistência à tração e à compressão, para os concretos convencionais, apresenta uma variação, conforme Mehta \& Monteiro (2008), entre 10 e 11\% e, nos protótipos testados, essa relação média se apresentou em 33,05\%, com variações entre 28,01\% (concreto com 50\% de Pinus spp. in natura) e 40,39\% (concreto com 50\% de Pinus spp. AF48H e 30\% de CCA). Tais relações se devem às características físicas e mecânicas do agregado lignocelulósico empregado como substituto parcial do agregado miúdo mineral convencional. Essa constatação é muito importante, pois o incremento verificado na resistência à compressão do material pode reduzir a incidência de patologias das construções, principalmente as motivadas por fissuração por tração e/ou absorção de pequenas deformações. Os valores médios determinados para a resistência à tração dos concretos dos blocos protótipos e de referência são apresentados na Figura 6.

Tabela 7. Resistência à tração dos blocos protótipos.

Table 7. Tension strength of prototypes blocks.

\begin{tabular}{lcc}
\multicolumn{1}{c}{ Blocos/composição } & \multicolumn{2}{c}{ Resistência à tração } \\
\cline { 2 - 3 } Concreto T $1: 10^{1}$ & RT 28 dias $(\mathbf{M P a})$ & CV (\%) \\
\hline PN $^{2}$ & $1,73^{\mathrm{AB}}$ & 4,88 \\
\hline PNCC $^{3}$ & $1,23^{\mathrm{D}}$ & 5,44 \\
PNAFCC $^{4}$ & $1,59^{\mathrm{C}}$ & 5,48 \\
PNAF SA $^{5}$ & $1,70^{\mathrm{BC}}$ & 4,48 \\
PNAFCV $^{6}$ & $1,62^{\mathrm{BC}}$ & 3,35 \\
PNAFCCA $^{7}$ & $1,71^{\mathrm{B}}$ & 5,54 \\
\hline
\end{tabular}

Letras diferentes denotam diferenças estatísticas entre as médias nas colunas no nível de 95\% de confiança; CV: Coeficiente de Variação; Concreto T 1:101: Concreto convencional para blocos traço 1:10; PN $^{2}: 50 \%$ de Pinus spp. in natura; PNCC $: 50 \%$ de Pinus spp. in natura $+4,5 \% \mathrm{CaCl}_{2} ; \mathrm{PNAFCC}^{4}: 50 \%$ de Pinus spp. in natura com 48 horas de tratamento em água fria $+4,5 \% \mathrm{CaCl}_{2}$; PNAFSA $^{5}: 50 \%$ de Pinus spp. in natura com 48 horas de tratamento em água fria $+10 \%$ de Sílica Ativa e 0,60\% de Superplastificante; PNAFCV 6 : $50 \%$ de Pinus spp. in natura com 48 horas de tratamento em água fria + 5\% de Cinza Volante; PNAFCCA $70 \%$ de Pinus spp. in natura com 48 horas de tratamento em água fria $+30 \%$ de Cinza de Casca de Arroz $+2,2 \%$ de Superplastificante.



Figura 6. Resistência à tração.

Figure 6. Tension strength. 


\section{CONCLUSÕES}

Com base nos resultados obtidos nesta pesquisa, pode-se concluir que:

- O sistema tradicional de blocos de concreto convencional se mostrou viável para a produção de blocos de concreto com substituições parciais do agregado miúdo mineral convencional (areia) pelo resíduo de Pinus spp. e de substituições parciais do cimento Portland, pelos três materiais cimentícios alternativos utilizados (Ś́lica Ativa, Cinza Volante e Cinza de Casca de Arroz);

- Os resultados determinados para as características físicas e mecânicas dos blocos protótipos demonstram a viabilidade técnica da utilização do resíduo de Pinus spp., como substituição parcial do agregado miúdo mineral para a produção de blocos de concreto para uso em alvenarias estruturais;

- A utilização de $\mathrm{CaCl}_{2} \cdot 2 \mathrm{H}_{2} \mathrm{O}$ como aditivo acelerador de pega propiciou ganhos significativos de resistência dos blocos protótipos em que o agregado miúdo mineral convencional foi substituído pelo resíduo de Pinus spp. nos concretos sem substituições do cimento pelos materiais cimentícios alternativos;

- A utilização do pré-tratamento do resíduo de Pinus spp. em água fria por 48 horas propiciou aumentos significativos da resistência à compressão dos blocos quando comparados aos blocos produzidos com resíduo de Pinus spp. sem tratamento, mesmo com uso do aditivo acelerador de pega $\left(\mathrm{CaCl}_{2} \cdot 2 \mathrm{H}_{2} \mathrm{O}\right)$;

- Os três materiais cimentícios alternativos utilizados se mostraram eficientes, pois propiciaram melhorias das propriedades físicas e mecânicas dos blocos protótipos, quando comparados aos blocos produzidos também com substituição parcial do agregado miúdo mineral pelo resíduo de Pinus spp., sem essas substituições;

- Diante dos limites estabelecidos pela normalização brasileira, os blocos produzidos com substituição parcial de 50\% em volume do agregado miúdo mineral convencional pelo resíduo de Pinus spp. apresentaram características físicas e mecânicas que possibilitam sua utilização em alvenarias estruturais, para a construção de edifícios de até cinco pavimentos;

- A utilização do resíduo de Pinus spp. como substituto ao agregado miúdo convencional reduziu a condutividade térmica média do concreto produzido, o que proporciona reflexos importantes para o desempenho dos blocos quanto ao conforto térmico dos ambientes construídos.

\section{STATUS DA SUBMISSÃO}

Recebido: 25/08/2011

Aceito: 26/09/2011

Resumo publicado online: 27/09/2011

Artigo completo publicado: 30/09/2011

\section{AUTOR(ES) PARA CORRESPONDÊNCIA}

\section{Adauto José Miranda de Lima}

Departamento Acadêmico de Construção Civil, Universidade Tecnológica Federal do Paraná UTFPR, Av. Sete de Setembro, 3165, Rebouças, CEP 80230-901, Curitiba, PR, Brasil

e-mail: adautojm@utfpr.edu.br

\section{Setsuo Iwakiri}

Departamento de Engenharia e Tecnologia Florestal, Universidade Federal do Paraná - UFPR,

Rua XV de Novembro, 1299, Centro, CEP 80060-000, Curitiba, PR, Brasil

e-mail: setsuo@ufpr.br

\section{REFERÊNCIAS}

Aïtcin PC. Concreto de Alto Desempenho. São Paulo: Editora PINI; 2000.

Associação Brasileira de Normas Técnicas - ABNT. NBR 15220-4. Desempenho térmico de edificações. Rio de Janeiro: ABNT; 2005.

American Society for Testing and Materials - ASTM. ASTM C 177-04: Standard test method for steady-state heat flux measurements and thermal transmission properties by means of the guarded-hot-plate apparatus. Pennsylvania: ASTM; 2008a.

American Society for Testing and Materials - ASTM. ASTM C 1170-08: Standard test method for determining consistency and density of roller-compacted concrete using a vibrating table.Pennsylvania: ASTM; 2008b.

Associação Brasileira da Indústria de Madeira Processada Mecanicamente. Estudo Setorial 2008. Availablefrom: http://www.abimci.com.br.

Associação Brasileira de Cimento Portland - ABCP. Produção de Blocos de Concreto para Alvenaria; Prática Recomendada. São Paulo, Boletim Técnico 107, ABCP, 1985, 12p. 
Associação Brasileira de Normas Técnicas - ABNT. NBR-5733: Cimento Portland de alta resistência inicial. Rio de Janeiro: ABNT; 1991a.

Associação Brasileira de Normas Técnicas- ABNT.NBR5736: Cimento Portland pozolânico. Rio de Janeiro: ABNT; 1991b.

Associação Brasileira de Normas Técnicas - ABNT. NBR-11578: Cimento Portland composto. Rio de Janeiro: ABNT;1991c.

Associação Brasileira de Normas Técnicas - ABNT. NBR-7222: Determinação da resistência à tração por compressão diametral de corpos-de-prova cilíndricos. Rio de Janeiro: ABNT; 1994.

Associação Brasileira de Normas Técnicas - ABNT. NBR-7215: Cimento Portland - Determinação da resistência à compressão. Rio de Janeiro: ABNT; 1997b.

Associação Brasileira de Normas Técnicas - ABNT. NBR-13956: Sílica ativa para uso em cimento Portland, concreto, argamassa e pasta de cimento Portland Especificação. Rio de Janeiro: ABNT;1997d.

Associação Brasileira de Normas Técnicas - ABNT. NBR-9479: Argamassa e concreto - Câmaras úmidas e tanques para cura de corpos-de-prova. Rio de Janeiro; $2006 a$.

Associação Brasileira de Normas Técnicas - ABNT. NBR-9778: Argamassas e concretos endurecidos Determinação da absorção de água, índice de vazios e massa específica. Rio de Janeiro: ABNT;2006b.

Associação Brasileira de Normas Técnicas - ABNT. NBR-6136: Blocos vazados de concreto simples para alvenaria - Requisitos. Rio de Janeiro: ABNT; 2008a.

Associação Brasileira de Normas Técnicas - ABNT. NBR-12118: Blocos vazados de concreto simples para alvenaria - Métodos de ensaio. Rio de Janeiro: ABNT;2008c.

Camões A. Betões de elevado desempenho com elevado volume de cinzas volantes. Minho, Portugal: Editora Universidade do Minho; 2005.

Carmo JBM.Análise comparativa do desempenho de concretos compostos com sílica ativa e metacaulimface a resistência mecânica e a viabilidade econômica. Curitiba: Universidade Federal do Paraná; 2006.

Dafico DA, Prudêncio Júnior LR. Um estudo visando a identificação das condições de queima da casca de arroz para a produção de pozolana branca. In: Anais doIX Encontro Nacional de Tecnologia do Ambiente Construído; 2002; Foz do Iguaçu. Foz do Iguaçu; 2002. p. 1671-1680.

Ferreira Júnior S. Produção de Blocos de Concreto para Alvenaria; Prática Recomendada. São Paulo: ABCP; 1985.
Freitas Júnior JA. Estudo comparativo de métodos de dosagem para concretos de alta resistência com o uso de materiais disponíveis na região metropolitana de Curitiba [dissertação]. Curitiba: Universidade Federal do Paraná; 2005.

Grandi SL. Alvenaria estrutural: a memória de um sistema construtivo. Prisma soluções construtivas com blocos de concreto2002; 5:21-23.

Instituto Brasileiro de Geografia e Estatística - IBGE. Produção Mundial de Arroz. Available from: http:// www.ibge.gov.br.

International Organization for Standardization - ISO. ISO 8302: Themal insulation - Determination of steady-state thermal resistance and related properties Guarded hot plate apparatus. ISO; 1991.

Isaia GC. Concreto: Ensino, Pesquisa e Realizações. São Paulo: IBRACON; 2005. v. 2.

Isaia GC. Materiais de construção civil e princípios de ciência e engenharia de materiais. São Paulo: IBRACON; 2007.

John VM, Agopyan V. Materiais reforçados com fibras vegetais. In: Anais do Simpósio Internacional de Materiais Reforçados com Fibras para a Construção Civil; 1993; São Paulo. São Paulo: EPUSP; 1993. p. 2938.

Lima AJM. Utilização de resíduo de Pinus spp e materiais cimentícios alternativos para produção de blocos para alvenaria estrutural [tese]. Curitiba: Universidade Federal do Paraná; 2009.

Medeiros JS. Alvenaria estrutural não armada de blocos de concreto: Produção de componentes e parâmetros de projeto [dissertação]. São Paulo: Escola Politécnica, Universidade de São Paulo; 1993.

Mehta PK, Monteiro PJM. Concreto: estrutura, propriedades e materiais. São Paulo: IBRACON; 2008.

Mendes SES. Estudo experimental de concreto de alto desempenho utilizando agregados graúdos disponíveis na região metropolitana de Curitiba [dissertação]. Curitiba: Universidade Federal do Paraná, 2002.

Moslemi AA. Inorganic-bonded wood composites: From sludge to siding. Journal of Forestry 1993; 91(11):27-29.

Moslemi AA. Wood-cement panel products: coming of age. In:Proceedings of the1st Inorganic Bonded Fiber Composites Symposium; 1989; USA.Forest Products Research Society; 1989. p. 12-18.

Neville AM. Propriedades do concreto. 2rd ed. São Paulo: Ed. PINI; 1997.

Oliveira CTA. Água do poro de pastas de cimento de escória [tese]. São Paulo: Escola Politécnica, Universidade de São Paulo; 2000. 
Pouey MTF.Beneficiamentos da cinza de casca de arroz residual com vistas à produção de cimento composto e/ou pozolânico[tese]. Porto Alegre: Escola de Engenharia, Universidade Federal do Rio Grande do Sul; 2006.

Ramirez-Coretti A, Eckelman CA. Wolf RW. Inorganicbonded composite wood panel systems for low-cost housing: a Central American perspective.Forest Products Journal 1998;48(4):62-68.

Santos S. Estudo da viabilidade de utilização de cinza de casca de arroz residual em argamassas e concretos [dissertação]. Florianópolis: Universidade Federal de Santa Catarina; 1997.
Semple KE, Evans PD. Wood-cement composites Suitability of Western Australian mallee eucalypt, blue gum and melaleucas. Kingston: Rural Industries Research and Development Corporation Kingston; 2004.

Sousa JGG. Contribuição ao estudo da relação entre propriedades e proporcionamento de blocos de concreto - aplicação ao uso de entulho como agregado reciclado [dissertação]. Brasília:Universidade de Brasília; 2001.

Tango CES. Blocos de concreto: dosagem, produção e controle de qualidade. São Paulo: IPT; 1984. 